\title{
Inflammation, oxidative stress, and glial cell activation characterize stellate ganglia from humans with electrical storm
}

\author{
Olujimi A. Ajijola, ${ }^{1,2}$ Donald B. Hoover, ${ }^{3,4}$ Thomas M. Simerly, ${ }^{3,4}$ T. Christopher Brown, \\ Jane Yanagawa, ${ }^{5}$ Reshma M. Biniwale, ${ }^{6}$ Jay M. Lee, ${ }^{5}$ Ali Sadeghi, ${ }^{6}$ Negar Khanlou, ${ }^{7}$ \\ Jeffrey L. Ardell, ${ }^{1,2}$ and Kalyanam Shivkumar ${ }^{1,2}$ \\ 'UCLA Cardiac Arrhythmia Center and 'UCLA Neurocardiology Research Center of Excellence, University of California, \\ Los Angeles, California, USA. ${ }^{3}$ Department of Biomedical Sciences, ${ }^{4}$ Center for Inflammation, Infectious Disease, and \\ Immunity, Quillen College of Medicine, East Tennessee State University, Johnson City, Tennessee, USA. Departments of \\ ${ }^{5}$ Thoracic, and ${ }^{6}$ Cardiac Surgery, and ${ }^{7}$ Pathology, University of California, Los Angeles, California, USA.
}

BACKGROUND. Neuronal remodeling in human heart disease is not well understood.

METHODS. Stellate ganglia from patients with cardiomyopathy (CMY) and refractory ventricular arrhythmias undergoing cardiac sympathetic denervation $(n=8)$, and from organ donors with normal hearts $(n=8)$ collected at the time of organ procurement were compared. Clinical data on all subjects were reviewed. Electron microscopy (EM), histologic, and immunohistochemical assessments of neurotransmitter profiles, glial activation and distribution, and lipofuscin deposition, a marker of oxidative stress, were quantified.

RESULTS. In CMY specimens, lipofuscin deposits were larger, and present in more neurons $\mathbf{2 6 . 3 \%}$ $\pm 6.3 \%$ vs. $16.7 \% \pm 7.6 \%, P<0.043$ ), than age-matched controls. EM analysis revealed extensive mitochondrial degeneration in CMY specimens. T cell (CD3+) infiltration was identified in $60 \%$ of the CMY samples, with one case having large inflammatory nodules, while none were identified in controls. Myeloperoxidase-immunoreactive neutrophils were also identified at parenchymal sites distinct from inflammatory foci in CMY ganglia, but not in controls. The adrenergic phenotype of pathologic samples revealed a decrease in tyrosine hydroxylase staining intensity compared with controls. Evaluation of cholinergic phenotype by staining for the vesicular acetylcholine transporter revealed a low but comparable number of cholinergic neurons in ganglia from both groups and demonstrated that preganglionic cholinergic innervation was maintained in CMY ganglia. S100 staining (a glial cell marker) demonstrated no differences in glial distribution and relationship to neurons; however, glial activation demonstrated by glial fibrillary acidic protein (GFAP) staining was substantially increased in pathologic specimens compared with controls.

CONCLUSIONS. Stellate ganglia from patients with CMY and arrhythmias demonstrate inflammation, neurochemical remodeling, oxidative stress, and satellite glial cell activation. These changes likely contribute to excessive and dysfunctional efferent sympathetic tone, and provide a rationale for sympathectomy as a treatment for arrhythmias in this population.

Role of funding source: Support for $\mathrm{OAA}, \mathrm{DBH}$, and KS, and materials and supplies used in this study.

Conflict of interest: The authors have declared that no conflict of interest exists.

Submitted: May 18, 2017

Accepted: August 8, 2017

Published: September 21, 2017

Reference information:

JCl Insight. 2017;2(18):e94715.

https://doi.org/10.1172/jici.

insight. 94715

FUNDING. This work was made possible by support from NIH grants HL125730 to OAA, GM107949 to DBH, and HL084261 and OT2OD023848 to KS.

\section{Introduction}

Neuroendocrine activation is central to the pathogenesis of heart disease. The influence of excess sympathetic activation on cardiovascular dysfunction, arrhythmias, and sudden death is well established $(1,2)$. Modulation of adrenergic signaling via pharmacologic and interventional approaches (3-5) is emerging as a major therapeutic strategy in managing ischemic (ICM) and nonischemic cardiomyopathy (NICM), and arrhythmias.

Functional and structural remodeling of sympathetic neurons in stellate ganglia (SGNs) of animals with ICM and NICM suggest that cardiac injury and the resulting chronic neurohormonal activation 
Table 1. Characteristics of control and surgical subjects

\begin{tabular}{|c|c|c|c|c|c|c|c|c|c|c|}
\hline Group & Age & Sex & Weight (kg) & Height & $\begin{array}{l}\text { Cause of Death or } \\
\text { Cardiomyopathy }\end{array}$ & CAD & LV EF & Comorbidities & VT Type & $\begin{array}{c}\text { Myocardial } \\
\text { Scar }\end{array}$ \\
\hline CMY & 18 & M & 68 & $6^{\prime} 3^{\prime \prime}$ & ARVC & No & $33 \%$ & None & MMVT + VF & Yes \\
\hline CMY & 26 & $M$ & 101 & $6^{\prime} 3^{\prime \prime}$ & dTGA & No & $40 \%$ & Th Outlet Syndrome & MMVT & Yes \\
\hline CMY & 31 & M & 64 & $5^{\prime} 9^{\prime \prime}$ & NICM & No & $10 \%$ & $\begin{array}{l}\text { AF, Asthma, CKD, } \\
\text { Hypothyroidism }\end{array}$ & MMVT + VF & No \\
\hline CMY & 32 & $M$ & 75 & $5^{\prime} 4^{\prime \prime}$ & Structurally normal & No & $55 \%$ & None & VF & No \\
\hline CMY & 36 & $M$ & 81 & $5^{\prime} 7^{\prime \prime}$ & NICM & No & $25 \%$ & AF, AVR, MVR, CKD & MMVT & Yes \\
\hline CMY & 37 & M & 81 & $5^{\prime} 10^{\prime \prime}$ & Sarcoidosis & No & $25 \%$ & None & MMVT & Yes \\
\hline CMY & 37 & M & 104 & $6^{\prime}$ & HCM & No & $40 \%$ & $\mathrm{AF}$ & MMVT & Yes \\
\hline CMY & 38 & $\mathrm{~F}$ & 139 & $5^{\prime} 7^{\prime \prime}$ & NICM & No & $45 \%$ & AF, HTN, Obesity, OSA & $\begin{array}{l}\text { MMVT + } \\
\text { PMVT }\end{array}$ & Yes \\
\hline CMY & 43 & $M$ & 127 & $5^{\prime} 7^{\prime \prime}$ & NICM & No & $55 \%$ & None & PMVT & No \\
\hline CMY & 45 & M & 113 & 5'10" & NICM & No & $50 \%$ & DVT & MMVT + VF & Yes \\
\hline CMY & 50 & $\mathrm{~F}$ & 79 & $5^{\prime} 3^{\prime \prime}$ & Chagas disease & No & $25 \%$ & HTN & MMVT & Yes \\
\hline CMY & 58 & $\mathrm{~F}$ & 75 & $5^{\prime} 4^{\prime \prime}$ & NICM & No & $51 \%$ & COPD & PMVT & No \\
\hline CMY & 63 & M & 94 & $6^{\prime}$ & NICM & No & $45 \%$ & DM, HTN, HL & MMVT & No \\
\hline CMY & 64 & M & 80 & $6^{\prime}$ & Mixed & Yes & $25 \%$ & AF, HTN, HL & MMVT & Yes \\
\hline CMY & 65 & M & 83 & $6^{\prime} 3^{\prime \prime}$ & Ischemic & Yes & $35 \%$ & AF, CKD, HTN & $\begin{array}{l}\text { MMVT + } \\
\text { PMVT }\end{array}$ & Yes \\
\hline CMY & 69 & $M$ & 89 & $5^{\prime} 6^{\prime \prime}$ & Mixed & Yes & $25 \%$ & AF, HTN & MMVT & Yes \\
\hline Control & 16 & $\mathrm{~F}$ & 50 & $5^{\prime} 4^{\prime \prime}$ & Head trauma & No & $65 \%$ & - & - & - \\
\hline Control & 22 & M & 114 & $6^{\prime} 0^{\prime \prime}$ & Intracranial hemorrhage & No & $70 \%$ & - & - & - \\
\hline Control & 24 & $\mathrm{~F}$ & 85 & $5^{\prime} 5^{\prime \prime}$ & Long QT & No & $65 \%$ & - & - & - \\
\hline Control & 24 & $\mathrm{~F}$ & 45 & $5^{\prime} 1^{\prime \prime}$ & Anoxic brain Injury & No & & - & - & - \\
\hline Control & 27 & M & 71 & $5^{\prime} 7^{\prime \prime}$ & Head trauma & No & $65 \%$ & - & - & - \\
\hline Control & 30 & M & 84 & $6^{\prime} 2^{\prime \prime}$ & Intracranial hemorrhage & No & $70 \%$ & - & - & - \\
\hline Control & 36 & M & 81 & $6^{\prime} 2^{\prime \prime}$ & Anoxic brain injury & No & $64 \%$ & - & - & - \\
\hline Control & 36 & M & 86 & $5^{\prime} 6^{\prime \prime}$ & Intracranial hemorrhage & No & $65 \%$ & - & - & - \\
\hline
\end{tabular}

Demographics and cardiac substrate for the subjects included in the study. AF, atrial fibrillation; ARVC, arrhythmogenic right ventricular cardiomyopathy; AVR, aortic valve replacement; CAD, coronary artery disease; CKD, chronic kidney disease; CMY, cardiomyopathy; COPD, chronic obstructive pulmonary disease; DM, diabetes mellitus; dTCA, d-transposition of the great vessels; DVT, deep venous thrombosis; F, female; HCM, hypertrophic cardiomyopathy; HL, hyperlipidemia; HTN, hypertension; LV EF, left ventricular ejection fraction; M, male; MMVT, monomorphic ventricular tachycardia; MVR, mitral valve replacement; NICM, nonischemic cardiomyopathy; OSA, obstructive sleep apnea; PMVT, polymorphic ventricular tachycardia; Th, thoracic; VF, ventricular fibrillation.

induce alterations in SGNs that facilitate the enhanced sympathetic tone seen in these animal models (5, 6). Data in humans are, however, scarce. Morphological size of SGNs along with synaptic density are increased in patients with cardiomyopathy (CMY) and arrhythmias (3). Further, in patients with inherited channelopathies undergoing sympathectomy for recurrent ventricular arrhythmias, evidence of inflammation was demonstrated (7). The neurochemical properties of SGNs from humans with CMY and arrhythmias, in whom chronic sympathoexcitation is characteristic, remain unknown.

The goal of this study was to determine how severe cardiac pathology alters SGN neurochemistry, as well as satellite glial cell (SGC) distribution and functional state, by comparing stellate ganglia from patients with CMY and arrhythmias undergoing cardiac sympathetic denervation, with those from control subjects serving as heart and/or lung donors.

\section{Results}

Table 1 shows characteristics of the CMY and control subjects included in the study. The mean age of the control subjects in the study was $28 \pm 8$ years, while the mean age for the CMY subjects was $44.5 \pm 15.4$ years. In total, 8 donors and 16 CMY patients were included in the study.

Ganglia from CMY patients exhibit evidence of oxidative stress. Lipofuscin, which is formed and accumulates in response to aging, as well as oxidative stress $(8,9)$, is a marker that is readily apparent in H\&E-stained sections. Accordingly, we examined ganglia from control and CMY groups for lipofuscin accumulation. 

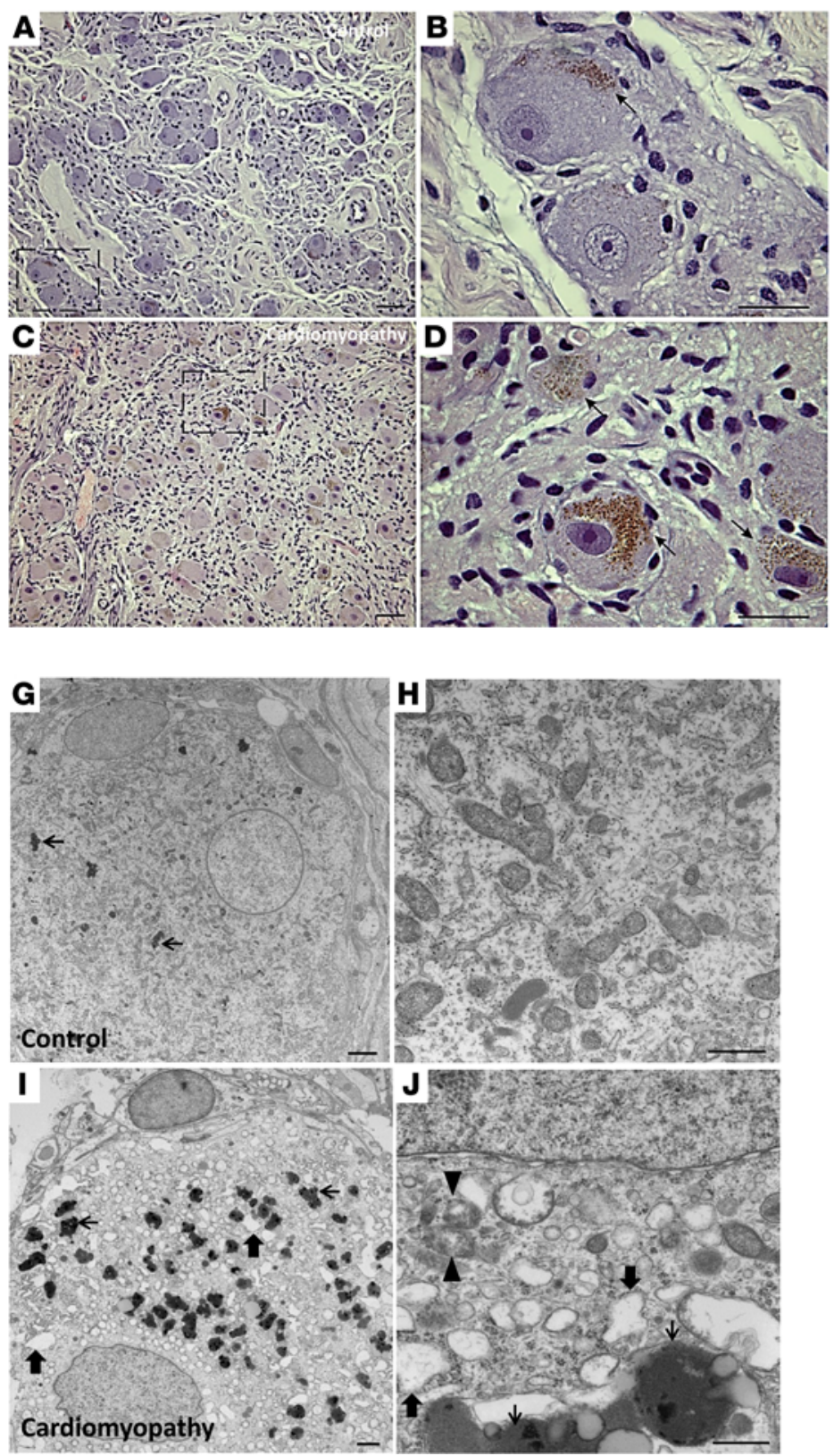

$\%$ Lipfuscin positive
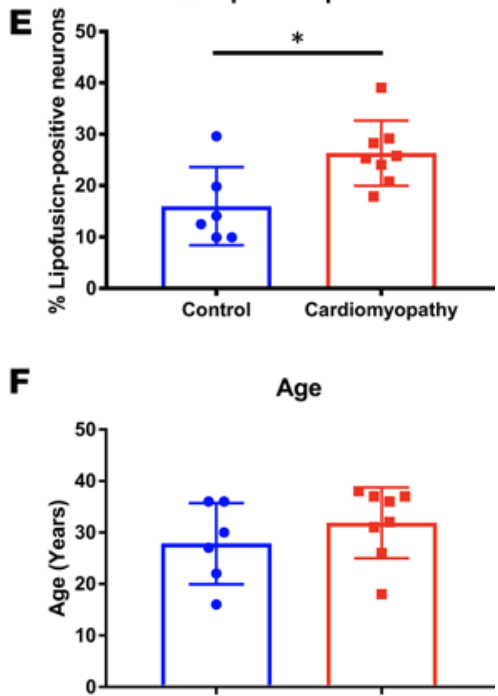

Figure 1. Oxidative stress in stellate ganglion neurons of patients with arrhythmias compared with controls. Representative images from stellate ganglia of (A and $\mathbf{B}$ ) control and (C and $\mathbf{D}$ ) cardiomyopathy patients showing accumulation of lipofuscin (arrows). Insets for panels $\mathbf{A}$ and $\mathbf{C}$ ( $\mathbf{B}$ and $\mathbf{D}$, respectively) show magnified neurons with lipofuscin accumulation. (E) Percentage of neurons showing lipofuscin accumulation in ganglia from control and cardiomyopathy patients (mean $\pm \mathrm{SD}, n=6$-8 per group, ${ }^{*} P=0.043$, Mann-Whitney test). (F) The age distribution of control and cardiomyopathy patients (mean \pm SD, $n=6$ and 8 for control and cardiomyopathy, respectively, $P=0.218$, Mann-Whitney test). Scale bars: $25 \mu \mathrm{m}$ (A and $\mathbf{C}$ ) and 50 $\mu \mathrm{m}$ (B and $\mathbf{D}) .(\mathbf{G}-\mathbf{J})$ Transmission electron microscopic images of stellate ganglion neurons from control ( $\mathbf{G}$ and $\mathbf{I}$ ) and cardiomyopathy ( $H$ and $\mathbf{J}$ ) subjects. Few oxidized lipid deposits exist in control (thin arrow), unlike neurons from cardiomyopathy subjects, which demonstrate numerous deposits, exhibit vacuolization (thick arrows), and mitochondrial degeneration (arrowheads). Scale bars: $2 \mu \mathrm{m}$ ( $\mathbf{G}$ and $\mathbf{H}$ ) and $500 \mathrm{~nm}$ ( $\mathbf{I}$ and $\mathbf{~})$.

Given the age dependence of lipofuscin deposition, age-matched control and CMY specimens were compared. As shown in Figure 1, a significantly greater percentage of neurons in CMY ganglia showed lipofuscin deposition compared with controls $(26.3 \% \pm 6.3 \%$ vs. $16 \% \pm 7.6 \%, P=0.043)$.

Oxidative stress is directly linked to mitochondrial function; therefore, we performed transmission electron microscopy (TEM) to examine whether mitochondria were structurally different between CMY and control subjects. TEM revealed extensive mitochondrial degeneration, with mitochondria in different phases of vacuolization identified (Figure 1, G-J). In addition, neurons from pathologic subjects showed numerous oxidized lipid deposits.

Leukocyte infiltration is a common feature of stellate ganglia from CMY patients. Further examination of $\mathrm{H} \& \mathrm{E}$-stained sections of left stellate ganglia (LSG) revealed prominent, dense clusters of leukocytes in one CMY patient (Figure 2). Leukocytes in this ganglion can be seen completely surrounding neurons (Figure 2, B and C), which stained intensely for tyrosine hydroxylase (TH, Figure 2D). Much smaller sites of leukocyte infiltration were found in ganglia from 2 other CMY patients, but none were evident in ganglia from the control patients. There was an overall incidence of $60 \%$ in CMY patients $(P<0.01)$. 

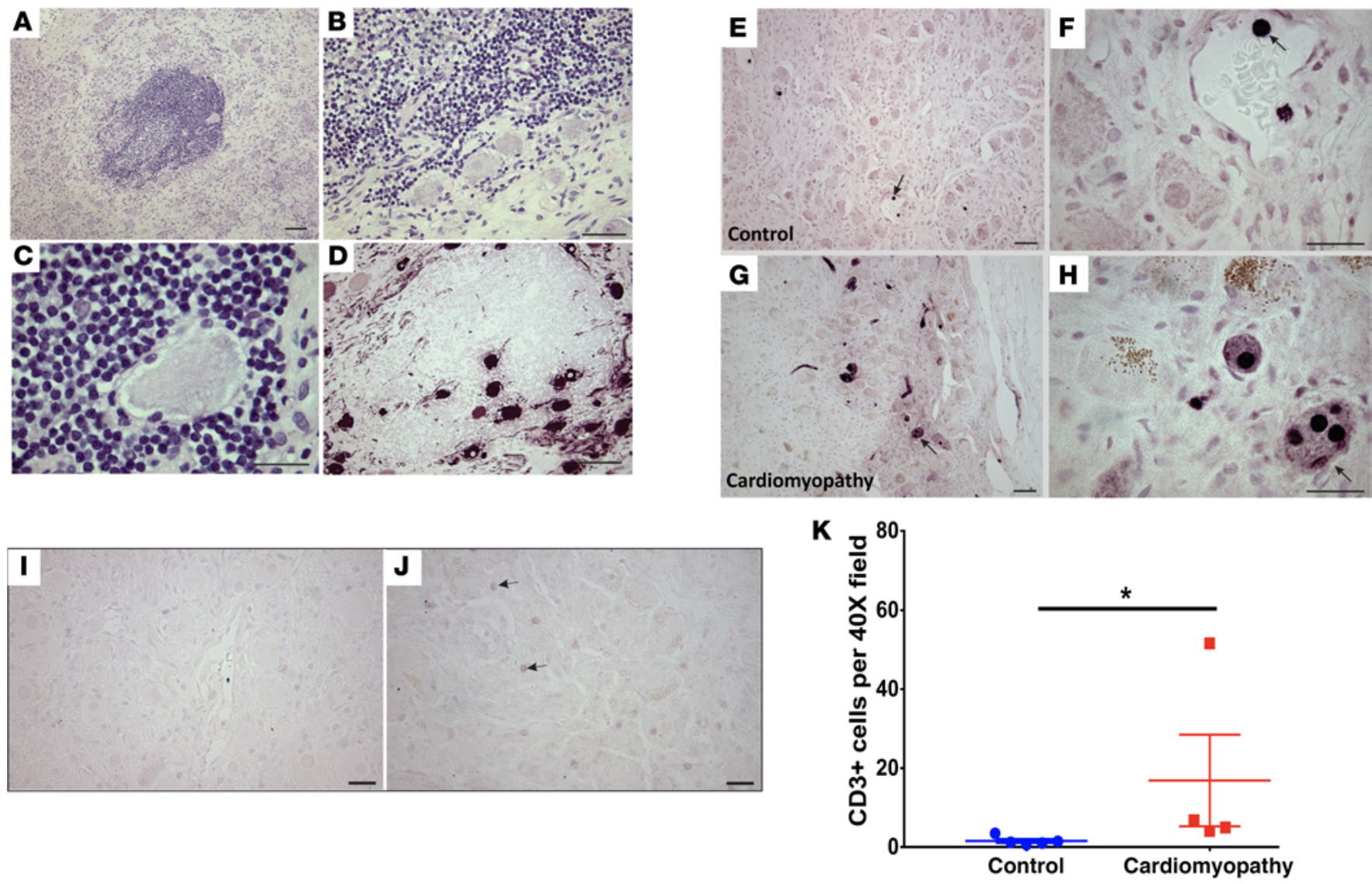

Figure 2. Leukocyte infiltration in stellate ganglia of patients with cardiomyopathy and arrhythmias. Images of extensive leukocyte infiltration of stellate ganglion of a cardiomyopathy patient are shown in panels A-D. (A and B) Images demonstrate a focus of inflammation at low and high magnifications, respectively, in $\mathrm{H} \& \mathrm{E}$-stained sections. ( $\mathbf{C}$ and $\mathbf{D}$ ) Images show the same inflammasome completely engulfing neurons. These neurons stained intensely for tyrosine hydroxylase (D). Scale bars: $100 \mu \mathrm{m}$ in A, $50 \mu \mathrm{m}$ in B and D, and $25 \mu \mathrm{m}$ in C. (E) Quantification of the incidence of inflammation in ganglia examined. (E-H) Brightfield photomicrographs showing immunostaining for myeloperoxidase in sections from control ( $\mathbf{E}$ and $\mathbf{F}$ ) and cardiomyopathy $(\mathbf{G}$ and $\mathbf{H})$ subjects' stellate ganglia. ( $\mathbf{E}$ and $\mathbf{F}$ ) Myeloperoxidase staining of control ganglion. Panel $\mathbf{F}$ shows a region of $\mathbf{E}$ at higher magnification ( $\times 100$, oil). Arrows in $\mathbf{E}$ and $\mathbf{F}$ indicate a labeled cell in a blood vessel. ( $\mathbf{G}$ and $\mathbf{H}$ ) Myeloperoxidase staining of surgical sample. Panel $\mathbf{H}$ shows a region of $\mathbf{G}$ at higher magnification ( $\times 100$, oil). Arrows in $\mathbf{G}$ and $\mathbf{H}$ indicate the same neuron with associated myeloperoxidase-labeled cells. Scale bars: $25 \mu \mathrm{m}$ in $\mathbf{F}$ and $\mathbf{H}$ and $50 \mu \mathrm{m}$ in $\mathbf{E}$ and $\mathbf{G}$. (I and J) CD3 immunoreactivity in a representative control and cardiomyopathy specimen, respectively. Arrows point to $C D 3^{+} T$ cells. Scale bars: $25 \mu \mathrm{m}$. (K) Graphical summary of the number of $C D 3^{+} T$ cells per high-power field (HPF) at $\times 40$ magnification. Mean $\pm \mathrm{SD}, n=5$ and 4 for control and cardiomyopathy, respectively. ${ }^{*} P=0.016$, Mann-Whitney test.

To further evaluate ganglionic inflammation, immunoreactivity to myeloperoxidase (MPO) was examined. MPO, a major component of neutrophil activity, is stored in large quantities in cytoplasmic granules and released in response to cellular activation. As shown in Figure 2, E-H, MPO immunoreactivity in control ganglia was only present in cells within blood vessels. In marked contrast, ganglia from CMY subjects demonstrated extracellular MPO deposits and MPO-immunoreactive neutrophils that were present in the parenchyma and associated with neurons. Further, few of the inflammatory infiltrates shown in Figure 2, $\mathrm{C}$ and D were MPO immunoreactive, suggesting that most are T cells (10). The presence of intraganglionic $\mathrm{T}$ cells was subsequently confirmed with CD3 staining (Figure 2, J-L).

SGNs from CMY patients exhibit a shift in adrenergic profiles. Stellate ganglia were stained for TH to identify adrenergic neurons. Adrenergic soma and nerve fibers were abundant in ganglia from both control and CMY patients (Figure 3, A and B), but the intensity of TH immunoreactivity in the cell bodies varied differently between groups (Figure 3C). In the control state, most neurons stained intensely for TH $(70.9 \% \pm$ $5.2 \%)$, compared with moderately stained $(26.6 \% \pm 5.6 \%)$, and TH-negative neurons $(4.5 \% \pm 1.9 \%, P<$ 0.001). CMY resulted in a significant shift in the distribution of relative intensity of TH staining, with moderately and intensely stained neurons being equivalent $(50 \% \pm 10.1 \%$ vs. $43.4 \% \pm 11.1 \%$, respectively, $P=$ 0.2 ). There was no significant difference between the percentage of TH-negative neurons in control versus CMY patients $(4.5 \% \pm 1.9 \%$ vs. $6.6 \% \pm 1.3 \%, P=0.2)$. 

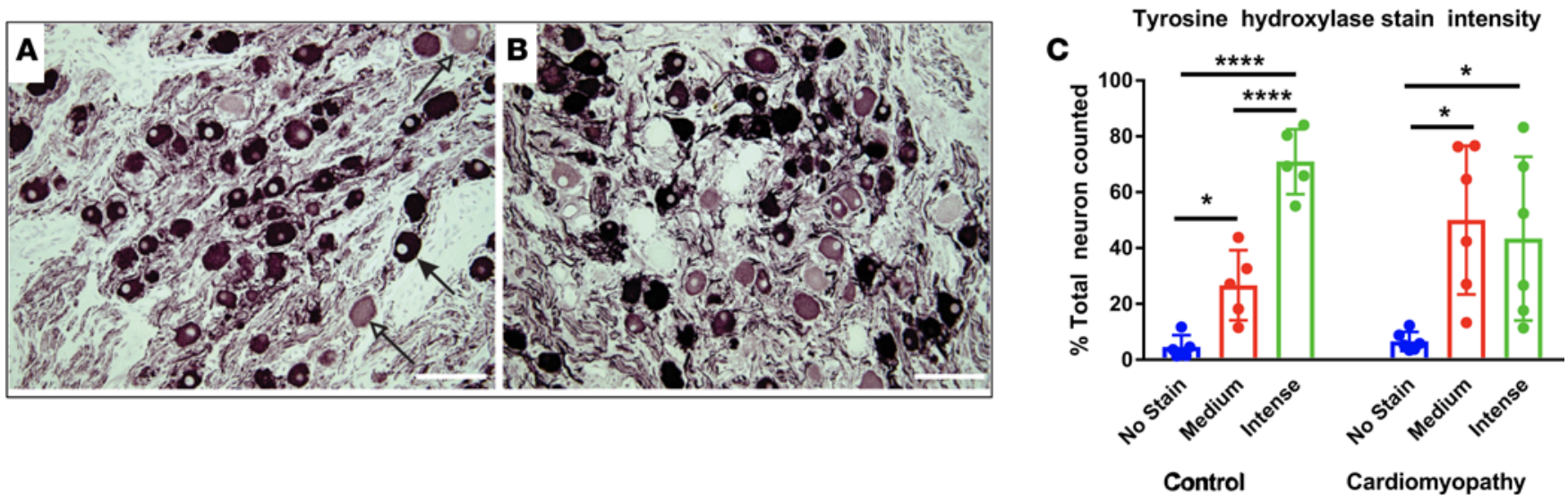

Figure 3. Shift in adrenergic profiles of stellate ganglion neurons from pathologic compared with control patients. Tyrosine hydroxylase-positive adrenergic neurons in stellate ganglia of control (A) and cardiomyopathy (CMY) patients (B). Fewer intensely stained neurons (solid arrow) can be identified in ganglia from CMY patients compared with controls, while medium-intensity neurons (open arrows) are increased in ganglia from CMY patients compared with controls. Scale bars: $100 \mu \mathrm{m}$. (C) The graphical summary of the neurochemical shift in adrenergic phenotype of the stellate ganglion neurons from both groups is shown (mean \pm SD). Data for each group were evaluated by 1-way ANOVA followed by Tukey's multiple comparisons test ( $n=5$ and 6 for control and cardiomyopathy, respectively). Horizontal lines indicate significant differences in percentage of neurons in specified staining categories. ${ }^{*} P<0.05$, ${ }^{* * * *} P<0.001$.

Cholinergic varicosities are not altered by CMY. Cholinergic elements within the stellate ganglia were identified by using an antibody against the vesicular acetylcholine transporter (VAChT). Extensive cholinergic varicosities were present in ganglia from both groups and frequently surrounded cell bodies that were revealed by background level of staining. The presence and distribution of these varicosities, which are preganglionic cholinergic fibers that provide excitatory input to the SGNs, did not differ between control and CMY subjects (Figure 4). Light to moderate staining for VAChT was evident in only a few SGNs (Figure 4), with no difference between controls and CMY patients.

Glial activation in pathological ganglia. SGCs in peripheral ganglia regulate the neuronal microenvironment and are important elements in neuronal function in health and disease (11-14). Using an antibody against the glia cell marker S100, we identified S100-positive satellite cells in close association with human SGNs (Figure 5, A and B). In fact, neurons were entirely enveloped by SGCs. The distribution and association of SGCs with SGNs were not significantly different between control and CMY subjects (Figure 5, C-F). S100-positive glial cells were also associated with nerve fibers in ganglia from control and CMY patients, with no obvious difference between groups.

We next examined glial cell activation in ganglia from CMY and control subjects using immunoreactivity to glial fibrillary acidic protein (GFAP) as a marker. As shown in Figure 6, compared with controls (Figure 6, A and B), ganglia from CMY subjects demonstrated substantially more GFAP immunoreactivity (Figure 6, C and D). GFAP-positive satellite cells were often observed surrounding neurons in ganglia from CMY patients but not in those from control patients (Figure 6, B and D). Likewise, ganglia from CMY patients had higher GFAP staining of glial cells associated with intraganglionic nerve fibers.

\section{Discussion}

Major findings. The major findings of the present study are: (a) SGNs from subjects with CMY and arrhythmias exhibit evidence of oxidative stress and a neurochemical shift in adrenergic but not cholinergic phenotype; (b) ganglia from CMY patients are characterized by leukocyte infiltration, the presence of $\mathrm{CD}^{+} \mathrm{T}$ cells, and MPO-positive neutrophils in the parenchyma; and (c) activation of glial cells, including perineuronal satellite cells and glial cells associated with intraganglionic nerve fibers. These findings provide direct evidence for inflammation and ongoing pathological remodeling within the stellate ganglia of CMY patients and support strategies aimed at mitigating adrenergic neurotransmission mediated by the stellate ganglia.

Oxidative stress and inflammation. The accumulation of lipofuscin and degeneration of mitochondria are indicators of cellular oxidative stress $(8,9,15)$. Increased metabolic activity is associated with increased rate of lipid peroxidation, an important step in lipofuscin formation and accumulation. In addition, oxidative stress is recognized to be a trigger for inflammation (16). Our findings of lipofuscin accumulation suggest increased activity of SGNs resulting in oxidative stress and mitochondrial dysfunction as a result. This 


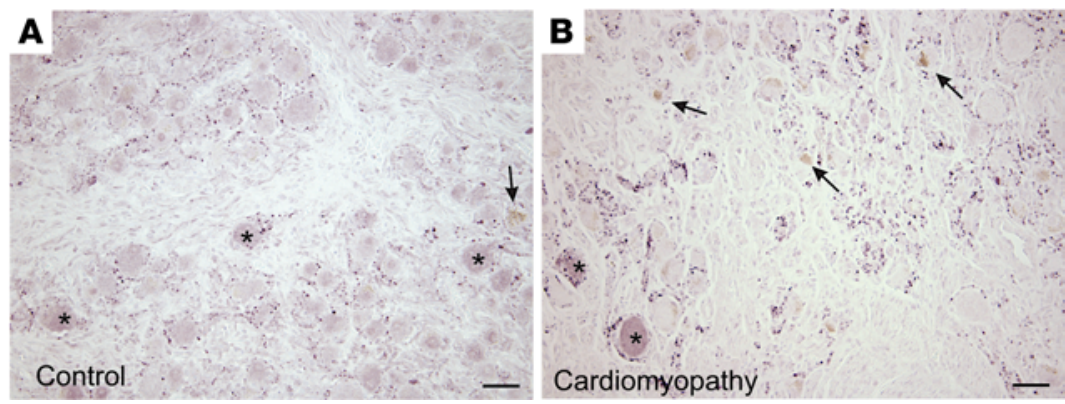

Figure 4. Neuronal cholinergic phenotypes are not different in control versus pathologic stellate ganglia. Bright-field photomicrographs showing immunostaining for the cholinergic marker, vesicular ACh transporter (VAChT), in sections from control (A) and surgical (B) samples of human stellate ganglia. Cholinergic varicosities appear as intense, punctate staining (purple) around neuronal cell bodies and, in some cases, present in the neuropil. Most stellate neurons showed a low but variable amount of background staining, but a few appeared darker than background (asterisk), suggesting the cholinergic phenotype. Visual inspection of stained sections from 4 control and 5 surgical ganglia revealed no obvious differences in VAChT staining between groups. Arrows indicate neurons that contain lipofuscin. Scale bars: $50 \mu \mathrm{m}$. pathophysiological process may contribute to local inflammation seen in the ganglia and evidenced by leukocyte infiltration and activation of SGCs. The trigger for increased neuronal activity and oxidative stress may be increased cardiac afferent neurotransmission, which increases efferent sympathetic tone via a spinal reflex mechanism (17). Other etiologies may include circulating neurohormonal signals such as angiotensin II $(18,19)$ or brainstem-mediated increases in efferent sympathetic outflow (20), all of which have been described in CMY and heart failure states. Cardiac inflammation and oxidative stress induced by repeated defibrillation might also contribute to ganglion pathology (21). The possible relationship between ganglion inflammation and cardiac arrhythmias was previously reported (22, 23); however, the suggested mechanisms were viral or unknown. We present evidence potentially implicating enhanced neuronal activity, and the oxidative

stress (resulting from this metabolic activity) as a trigger for inflammation. MPO, released from neutrophils (10), is an important modulator of both acute and chronic inflammation (24). Oxidants derived from MPO are known to interfere with cellular physiologic processes. The presence of MPO-containing neutrophils in association with neurons may suggest ongoing neuronal dysfunction and enhanced cardiac sympathetic instability, although an important factor to consider is the timing of ganglia collection in organ donors, which occurred following cessation of circulation in most cases.

Adrenergic profiles in patients with CMY and ventricular arrhythmias. Enhanced sympathetic tone, mediated in part by neurotransmission through stellate ganglia, is associated with the progression of cardiovascular pathogenesis and risk of arrhythmias and sudden death (1). The adrenergic marker $\mathrm{TH}$, which catalyzes the rate-limiting step in the synthesis of catecholamines, is produced in the cell body and transported to nerve terminals where norepinephrine is synthesized, stored, and released. The decrease in intensity of $\mathrm{TH}$ staining in soma of SGNs from CMY patients may appear counterintuitive; however, it likely represents mobilization of enzyme to the nerve terminals to support catecholamine generation and release. In addition to reported structural changes to neurons (25-27), the findings of the present study support enhanced activity in SGNs in patients with CMY and arrhythmias.

Cholinergic properties were not different between CMY and control patients. Cholinergic neurons make up a minority of SGNs, and are postulated to innervate non-cardiac structures of the upper thorax, for example sweat glands. Whether cholinergic neurons in the stellate ganglia have a role in cardiac neurotransmission is not clear. Studies in animal models have suggested that cholinergic remodeling occurs in cardiac disease. In a rodent model of nonischemic heart failure (28), sympathetic-to-cholinergic transdifferentiation of SGNs mediated by gp130 cytokines was reported. In a porcine model of ischemic CMY (6), fewer cholinergic SGNs were identified when compared with control animals. Although these findings in animal models of subacute pathology suggest that shifts in the neurotransmitter phenotype of SGNs can occur with pathology, cholinergic remodeling does not appear to be a feature of more severe and complex chronic CMY in humans.

SGCs closely associated with SGNs are activated by cardiac pathology. The microenvironment in which SGNs function is regulated by SGCs, which ensheathe the somata of the sympathetic neurons and the synaptic inputs to their dendrites (11). Accordingly, these glial cells are well placed to affect synaptic transmission and neuronal excitability. Several lines of evidence suggest that SGCs have bidirectional interactions with the neurons that they surround and with preganglionic nerve terminals. SGCs express ion channels (29), which provide a mechanism for regulation of the extracellular fluid composition, receptors for neurotransmitters (30) and neurotrophic factors (31), and they are able to release mediators that affect neurons (23). Furthermore, active control of the neuronal microenvironment by SGCs can contribute to disease (32). In the present study, we did not identify alterations in the distribution and arrangement of SGCs in stellate ganglia; however, many SGCs exhibited activation, as evidenced by strong GFAP immunoreactivity. To our knowledge, this represents the first report of glial activation in 

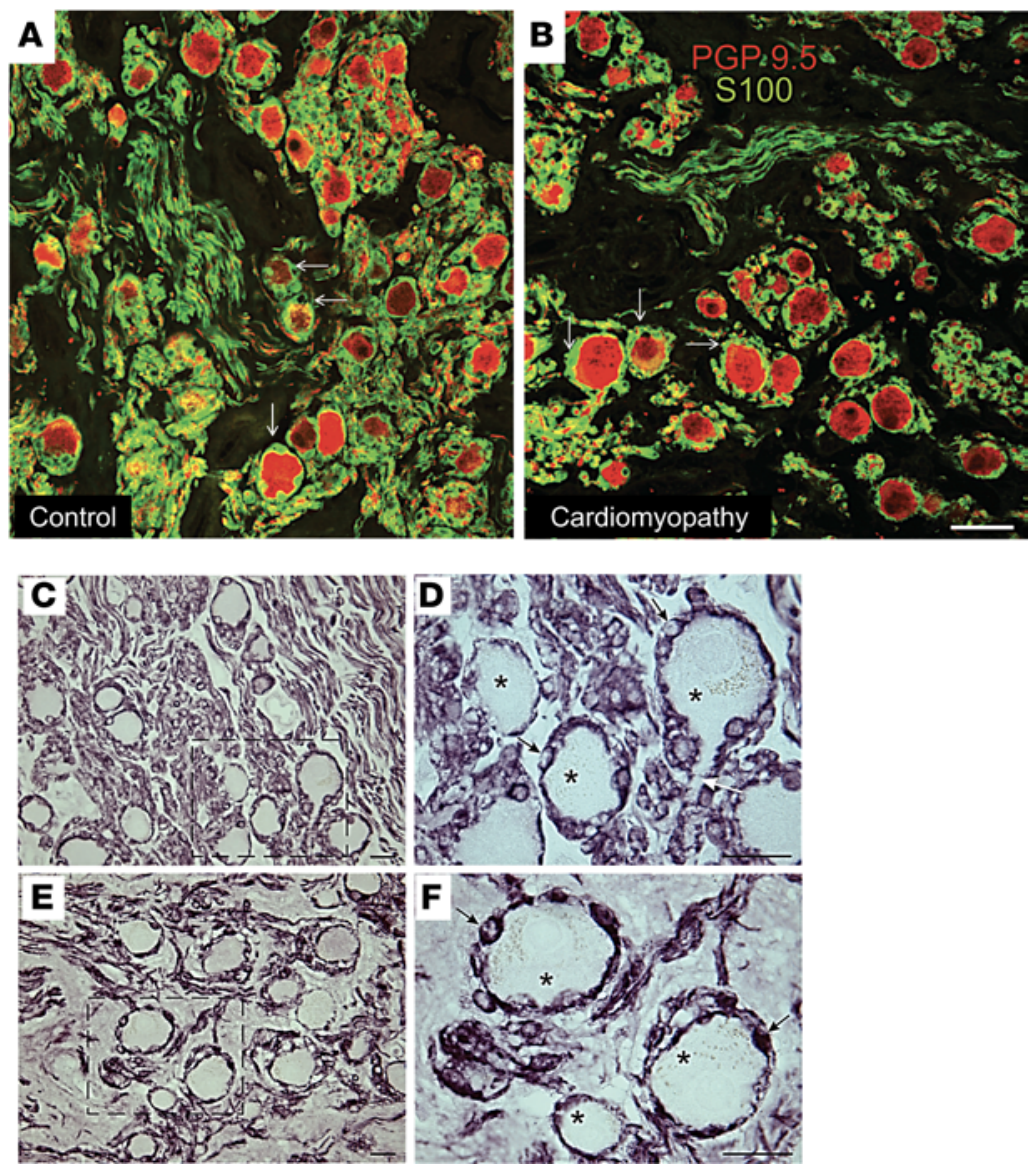

Figure 5. Association of satellite cells with stellate ganglion neurons. Confocal microscopic images of human stellate ganglion sections double labeled for the pan neuronal marker PGP9.5 (red) and the glial cell marker S100 (green). (A) Control ganglion. (B) Ganglion from a cardiomyopathy subject. In both panels, sympathetic neurons (red) are surrounded by green satellite glial cells (white arrows) The surrounding neuropil contains labeled nerve fibers and associated glial cells. Scale bar: $50 \mu \mathrm{m}$. (C-F) Brightfield photomicrographs showing immunostaining for $\mathbf{S 1 0 0}$ in sections from control (C and $\mathbf{D})$ and surgical (E and $\mathbf{F}$ ) samples of human stellate ganglia. (C and D) S100 staining of control ganglion. Region indicated by dashed box in panel $\mathbf{C}$ is shown at higher magnification $(\times 100$, oil) in panel $\mathbf{D}$. (E and $\mathbf{F}$ ) S100 staining of surgically removed ganglion. Region indicated by dashed box in panel $\mathbf{E}$ is shown at higher magnification $(\times 100$, oil) in panel F. 5100 staining is also associated with glial cells and their processes in the neuropil. There is no obvious difference in staining for control versus surgical samples. Asterisk, neuron; black arrow, satellite glial cell; white arrow, axon leaving neuron. Scale bars: $25 \mu \mathrm{m}$.

stellate ganglia of patients with CMY and arrhythmias. Taken together with the finding of inflammation and oxidative stress within stellate ganglia, this finding suggests that SGNs in patients with CMY and arrhythmias are under pathologic stress. It is not clear whether SGCs are reacting to such stress to maintain homeostatic balance or whether they are contributing to it. However, in the context of CMY, it is reasonable to speculate that SGC activation contributes to the pathophysiology. Systemic inflammation, a feature of chronic CMY and heart failure, and regional inflammation have both been demonstrated to activate SGCs and augment coupling of SGCs via gap junctions (33). Further, evidence has implicated increased coupling of SGCs to neuronal hyperexcitability (31), which could be a key factor in the pathophysiology of ventricular tachycardia in CMY patients.

Figure 6. Satellite glial cells from patients with arrhythmias and cardiomyopathy are activated. Bright-field photomicrographs showing immunostaining for GFAP in sections from control ( $\mathbf{A}$ and $\mathbf{B})$ and cardiomyopathy (C and $\mathbf{D}$ ) samples of human stellate ganglia. (A and $\mathbf{B}$ ) GFAP staining is sparse in control ganglion. Region indicated by dashed box in panel $\mathbf{A}$ is shown at higher magnification ( $\times 100$, oil) in panel B. (C and D) Strong GFAP staining of satellite glial cells and neuropil is evident in surgically removed ganglion. Region indicated by dashed box in panel $\mathbf{C}$ is shown at higher magnification $(\times 100$, oil) in panel $\mathbf{D}$. GFAP staining in the neuropil is most likely on glial cells and processes associated with axons. Asterisk, neuron; black arrow, satellite glial cell. Scale bars: $25 \mu \mathrm{m}$.
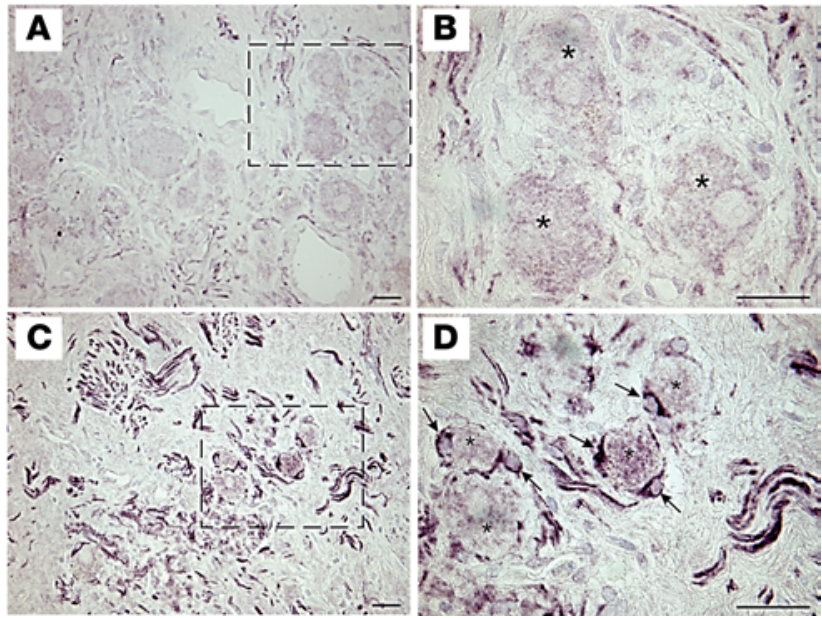


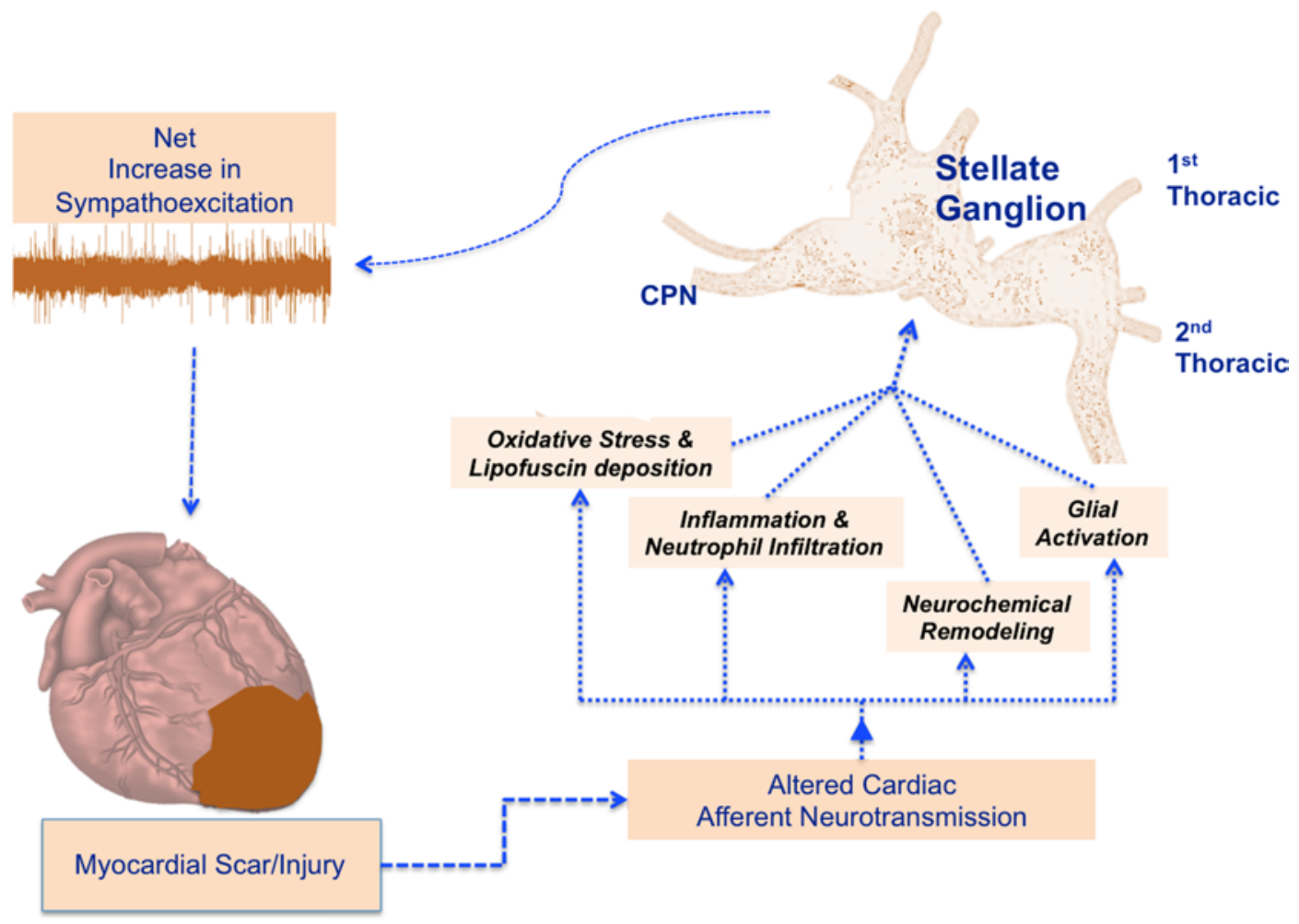

Figure 7. Potential pathways driving adverse remodeling within stellate ganglia and impact of efferent sympathetic neurotransmission. Schematic representation depicting the impact of myocardial injury on stellate ganglion neurons, inducing the alterations identified in this work. CPN, cardiopulmonary nerve.

Stellate ganglia as nexus points for neuromodulation. These findings support the use of denervation via partial resection of the stellate ganglion to control arrhythmias $(5,34,35)$ and to mitigate the progression of dilated CMY (36). Pathological changes within stellate ganglia that act to enhance neurotransmission create further electrical instability (37), increasing the risk of arrhythmias. Removal of a segment of the sympathetic chain may also eliminate these regions of the nervous system, which can potentially amplify sympathoexcitation.

Limitations. Although the sample size is small, the use of stellate ganglia from normal controls (noncadaveric) allowed uniform fixation across samples, which has not previously been performed. Further, entire ganglia were sectioned and studied for complete analyses. The small sample size prevented specific relation of changes within ganglia to specific clinical or pathological features in patients. The age of control and CMY subjects differed. This is unavoidable, as organ donors are typically young, healthy subjects. Ganglia from donors were typically harvested after cessation of circulation; hence, it is conceivable that the lack of neutrophils within control ganglia is related to the lack of circulation.

Conclusions. In summary, our study demonstrates, for the first time to our knowledge, that stellate ganglia from patients with CMY and arrhythmias exhibit oxidative stress, inflammation, neurochemical remodeling, and SGC activation (Figure 7). These findings suggest that stellate ganglion dysfunction is an important mediator of enhanced sympathetic neurotransmission associated with arrhythmias and support cardiac sympathetic denervation as an important neuromodulatory intervention for arrhythmia control.

\section{Methods}

\section{Specimen collection}

CMY specimens. Stellate ganglia $(n=8)$ were collected from CMY patients undergoing cardiac sympathetic denervation (the resection of the lower half of the stellate ganglion, and second through fourth paravertebral ganglia). Patients consented to provide the excised stellate ganglia for research. Histology slides from another group of $8 \mathrm{CMY}$ patients were also examined. These were patients with depressed left ventricular function and severe ventricular arrhythmias refractory to pharmacologic and invasive 
Table 2. List of antibodies, sources, and concentrations used

\begin{tabular}{lcc}
\hline Antibody & Concentration & Source \\
Anti-Tyrosine Hydroxylase & $1: 500$ & Polyclonal Rabbit Antibody, PelFreez, catalog P40101-150 \\
Anti-PGP9.5 & $1: 1,000$ & Polyclonal Guinea Pig Antibody, Neuromics, catalog GP1404 \\
Anti-Vesicular Acetylcholine Transporter & $1: 1,000$ & Polyclonal Rabbit Antibody, Synaptic Systems, catalog 139103 \\
Anti-Human Myeloperoxidase & $1: 1,000$ & Polyclonal Rabbit Antibody, Dako, catalog A0398 \\
Anti-S100 & $1: 250$ & Polyclonal Rabbit Antibody, Dako, catalog Z0311 \\
Anti-Glial Fibrillary Acidic Protein & $1: 1,000$ & Polyclonal Rabbit Antibody, Dako, catalog Z0334 \\
Donkey Anti-Rabbit $\left(2^{\circ}\right)$ Alexa Fluor 594 Conjugate & $1: 200$ & Polyclonal Rabbit, Thermo Fisher Scientific, catalog A-21207 \\
Goat Anti-Guinea Pig $\left(2^{\circ}\right)$ Alexa Fluor 594 Conjugate & $1: 200$ & Polyclonal Goat, Thermo Fisher Scientific, catalog A-11076 \\
Donkey Anti-Rabbit $\left(2^{\circ}\right)$ Alexa Fluor 488 Conjugate & $1: 200$ & Polyclonal Rabbit, Thermo Fisher Scientific, catalog A-21206 \\
Anti-CD3 (clone SP7) & $1: 50$ & Monoclonal Rabbit Antibody, Thermo Fischer Scientific, catalog MA1-90582
\end{tabular}

treatment strategies such as catheter ablation (Table 1). Detailed clinical information on these patients was also collected, such as demographics, medical history, and records of noninvasive and invasive clinic studies and assessments. Following excision, stellate ganglia were rapidly collected and placed immediately in fixative, as described below.

Control specimens. Stellate ganglia $(n=8)$ were collected from organ donors at the time of organ procurement with consent provided for research tissue collection. Use of these tissues was in accordance with institutional guidelines and was approved by the UCLA IRB. All available clinical information including age, gender, medical history, and cause of death were reviewed. Patients with cardiac or pulmonary diseases were excluded. Following removal of the heart and lungs for transplantation, stellate ganglia were rapidly collected and placed immediately in fixative, as described.

\section{Tissue sectioning and preparation}

LSG and right stellate ganglia (RSG) were evaluated. Samples were placed immediately into cold $10 \%$ phosphate-buffered formalin (Thermo Fisher Scientific) for 7 days, transferred to $70 \%$ ethanol, and subsequently embedded in paraffin.

Paraffin-embedded ganglia were sectioned at $5 \mu \mathrm{m}$ thickness using a Microm HM 310 microtome (Southeast Pathology). Sections were collected on charged slides, and prior to staining, they were deparaffinized by incubation at $58^{\circ} \mathrm{C}-60^{\circ} \mathrm{C}$ overnight followed by xylene washes and then rehydration in a graded ethanol series ending with water.

\section{Histology}

From each ganglion, 7-10 evenly spaced slides were stained with H\&E. These slides were used to identify regions containing the most neurons, neurons containing lipofuscin, and assess leukocyte infiltration.

\section{Immunohistochemistry}

Antigen retrieval was performed using heated sodium citrate buffer $(0.882 \mathrm{~g}$ per $300 \mathrm{ml}$ distilled water, $\mathrm{pH}$ 6.9, for 4 minutes), and washed 4 times in $0.1 \mathrm{M}$ phosphate-buffered saline (PBS, pH 7.3). Sections were permeabilized with $0.4 \%$ Triton X-100 (TX-100) in PBS containing $0.5 \%$ bovine serum albumin (BSA), then $1 \% \mathrm{H}_{2} \mathrm{O}_{2}$ in PBS. Slides were washed again in PBS, and blocked for 2 hours in PBS containing $5 \%$ normal donkey serum, $0.5 \%$ BSA, and $0.4 \%$ TX-100. Slides were then incubated overnight at room temperature in blocking buffer containing primary antibodies, and subsequently washed in PBS, followed by blocking for 2 hours. Subsequent steps varied depending on the detection technique. For fluorescence detection, slides were incubated with AlexaFluor-conjugated secondary antibodies (Table 2) for 2 hours and washed with PBS. Cover glasses were applied using Citifour AF1 mounting medium (Ted Pella Inc.) and sealed. Kits containing species-specific biotinylated secondary antibodies were used for detection by the ABC technique (Vector Laboratories). Bright-field slides were then dehydrated and cover glasses were applied using Cytoseal XYL mounting medium (Thermo Fisher Scientific). 


\section{Microscopy and image analysis}

Slides were viewed and photographed under bright field or fluorescence illumination using an Olympus BX41 microscope. Digital images were obtained using an Olympus Qcolor 3 camera. Select slides were further examined using confocal microscopy with a Leica SP8 confocal microscope (Leica Microsystems Inc.).

For quantitative analysis of lipofuscin-positive neurons and TH staining, we evaluated at least 3 sections from different levels within each ganglion and collected 6-7 images of $\times 40$ fields per section. Stereo Investigator software (MicroBrightField, Inc.) was used to identify and detect lipofuscin-positive neurons based on color. Staining of neurons for TH was graded as intense, moderate, or absent. In each case, different populations of neurons were labeled with a unique marker in Stereo Investigator and counted.

\section{TEM}

Each specimen measured approximately 7 to $10 \mathrm{~mm}$ in length. Cross-sections, perpendicular to the length, were obtained. Efforts were made to obtain a uniform $1 \times 1 \mathrm{~mm}$ specimen dimension per block. The tissue was fixed in $2.5 \%$ glutaraldehyde $/ 0.1 \mathrm{M}$ sodium phosphate buffer $\mathrm{pH} 7.4$ for 24 hours at $4^{\circ} \mathrm{C}$, followed by $1 \%$ osmium tetroxide in $0.1 \mathrm{M}$ sodium phosphate buffer for 2 hours. Each case yielded 3-6 sections. Tissue sections were then infiltrated by progressive composite of epon $(1: 1$ to $3: 1)$ in acetone and placed in embedding molds (Electron Microscopy Sciences, 70166, block shape). One-micron-thick methylene blue-stained thick sections were prepared from each block and reviewed. The most representative block was selected and the area of interest was designated for TEM grid preparation. The resin block was then trimmed and cut in 90- to 120 -nm thin sections and captured on TEM grids. These were stained with $4 \%$ uranyl acetate followed by lead citrate. The grids were allowed to dry in room temperature prior to examination in an FEI/PHILIPS EM208S transmission electron microscope. Multiple digital images at final print magnifications of $\times 3,690$ to $\times 59,100$ at $150 \mathrm{~mm}$ and direct magnification ranges of $\times 2,000$ to $\times 40,000$ were captured.

\section{Primary and secondary antibodies}

A list of the antibodies used and concentrations is provided in Table 2.

\section{Statistics}

Data are expressed as means \pm SD. Continuous variables were compared using a 2-tailed Student's $t$ test or ANOVA for normally distributed variables, and the Mann-Whitney test for variables not distributed normally. An adjusted $P$ value of less than or equal to 0.05 was considered statistically significant.

\section{Study approval}

The study was approved by the UCLA IRB. Written informed consent was provided by the patient or appropriate designee. For organ donors, consent for research was provided by the donor or appropriate signatory.

\section{Author contributions}

OAA, DBH, JLA, and KS designed the study. OAA, DBH, TMS, and TCB conducted experiments. OAA, JY, JML, RMB, AS, and NK acquired the tissue and data. OAA, DBH, TCB, TMS, and NK analyzed the data. OAA, DBH, JLA, and KS wrote the manuscript.

\section{Acknowledgments}

The authors wish to thank the tissue donors, without whom this study would not be possible. The authors also wish to acknowledge One Legacy and the California Transplant Donor Network (CTDN) for support in retrieving tissue. The authors also wish to thank Oh Jin Kwon, James Tollins, Msgana Tamrat, Hugo Perez, Biliet L. Ngang, and Jeffrey T. Michaels for invaluable contributions to this project. This work was made possible by support from NIH grants HL125730 to OAA, GM107949 to DBH, and HL084261 and OT2OD023848 to KS.

Address correspondence to: Olujimi A. Ajijola or Kalyanam Shivkumar, UCLA Cardiac Arrhythmia Center, UCLA Health System, David Geffen School of Medicine at UCLA, Suite 660, Westwood Boulevard, Los Angeles, California 90095-1679, USA. Phone: 310.206.6433; Email: oajijola@mednet.ucla.edu (O.A. Ajijola); kshivkumar@mednet.ucla.edu (K. Shivkumar). 
1. Shivkumar K, et al. Clinical neurocardiology defining the value of neuroscience-based cardiovascular therapeutics. $J$ Physiol (Lond). 2016;594(14):3911-3954.

2. Ardell JL, et al. Translational neurocardiology: preclinical models and cardioneural integrative aspects. J Physiol (Lond). 2016;594(14):3877-3909.

3. Schwartz PJ, Locati EH, Moss AJ, Crampton RS, Trazzi R, Ruberti U. Left cardiac sympathetic denervation in the therapy of congenital long QT syndrome. A worldwide report. Circulation. 1991;84(2):503-511.

4. Bourke T, et al. Neuraxial modulation for refractory ventricular arrhythmias: value of thoracic epidural anesthesia and surgical left cardiac sympathetic denervation. Circulation. 2010;121(21):2255-2262.

5. Ajijola OA, et al. Bilateral cardiac sympathetic denervation for the management of electrical storm. J Am Coll Cardiol. 2012;59(1):91-92.

6. Ajijola OA, et al. Remodeling of stellate ganglion neurons after spatially targeted myocardial infarction: Neuropeptide and morphologic changes. Heart Rhythm. 2015;12(5):1027-1035.

7. Rizzo S, et al. T-cell-mediated inflammatory activity in the stellate ganglia of patients with ion-channel disease and severe ventricular arrhythmias. Circ Arrhythm Electrophysiol. 2014;7(2):224-229.

8. Goyal VK. Lipofuscin pigment accumulation in human brain during aging. Exp Gerontol. 1982;17(6):481-487.

9. Brunk UT, Terman A. The mitochondrial-lysosomal axis theory of aging: accumulation of damaged mitochondria as a result of imperfect autophagocytosis. Eur J Biochem. 2002;269(8):1996-2002.

10. Pinkus GS, Pinkus JL. Myeloperoxidase: a specific marker for myeloid cells in paraffin sections. Mod Pathol. 1991;4(6):733-741.

11. Hanani M. Satellite glial cells in sympathetic and parasympathetic ganglia: in search of function. Brain Res Rev. 2010;64(2):304-327.

12. Ribeiro-Resende VT, Carrier-Ruiz A, Lemes RM, Reis RA, Mendez-Otero R. Bone marrow-derived fibroblast growth factor-2 induces glial cell proliferation in the regenerating peripheral nervous system. Mol Neurodegener. 2012;7:34.

13. Vaegter CB. Neurotrophins and their receptors in satellite glial cells following nerve injury. Neural Regen Res. 2014;9(23):2038-2039.

14. Zhou XF, et al. Satellite-cell-derived nerve growth factor and neurotrophin-3 are involved in noradrenergic sprouting in the dorsal root ganglia following peripheral nerve injury in the rat. Eur J Neurosci. 1999;11(5):1711-1722.

15. Sohal RS, Brunk UT. Lipofuscin as an indicator of oxidative stress and aging. Adv Exp Med Biol. 1989;266:17-26.

16. Salminen A, Ojala J, Kaarniranta K, Kauppinen A. Mitochondrial dysfunction and oxidative stress activate inflammasomes: impact on the aging process and age-related diseases. Cell Mol Life Sci. 2012;69(18):2999-3013.

17. Wang HJ, Wang W, Cornish KG, Rozanski GJ, Zucker IH. Cardiac sympathetic afferent denervation attenuates cardiac remodeling and improves cardiovascular dysfunction in rats with heart failure. Hypertension. 2014;64(4):745-755.

18. Xiao L, Haack KK, Zucker IH. Angiotensin II regulates ACE and ACE2 in neurons through p38 mitogen-activated protein kinase and extracellular signal-regulated kinase 1/2 signaling. Am J Physiol, Cell Physiol. 2013;304(11):C1073-C1079.

19. Zucker IH, Gao L. The regulation of sympathetic nerve activity by angiotensin II involves reactive oxygen species and MAPK Circ Res. 2005;97(8):737-739.

20. Lambert GW, et al. Increased central nervous system monoamine neurotransmitter turnover and its association with sympathetic nervous activity in treated heart failure patients. Circulation. 1995;92(7):1813-1818.

21. Moss AJ, Ryan DH, Yeaney GA. Ganglionitis and genetic cardiac arrhythmias: more questions than answers. Circ Arrhythm Electrophysiol. 2014;7(2):190-191.

22. James TN, Imamura K. Virus-like particles associated with intracardiac ganglionitis in 2 cases of sudden unexpected death. Jpn Heart J. 1981;22(3):447-454.

23. James TN, Zipes DP, Finegan RE, Eisele JW, Carter JE. Cardiac ganglionitis associated with sudden unexpected death. Ann Intern Med. 1979;91(5):727-730.

24. Nussbaum C, Klinke A, Adam M, Baldus S, Sperandio M. Myeloperoxidase: a leukocyte-derived protagonist of inflammation and cardiovascular disease. Antioxid Redox Signal. 2013;18(6):692-713.

25. Ajijola OA, et al. Extracardiac neural remodeling in humans with cardiomyopathy. Circ Arrhythm Electrophysiol. 2012;5(5):1010-1116

26. Docimo S, Piccolo C, Van Arsdale D, Elkowitz DE. Pathology-dependent histological changes of the left stellate ganglia: a cadaveric study. Clin Med Pathol. 2008;1:105-113.

27. Wood A, Docimo S, Elkowitz DE. Cardiovascular disease and its association with histological changes of the left stellate ganglion. Clin Med Insights Pathol. 2010;3:19-24.

28. Kanazawa $\mathrm{H}$, et al. Heart failure causes cholinergic transdifferentiation of cardiac sympathetic nerves via gp130-signaling cytokines in rodents. J Clin Invest. 2010;120(2):408-421.

29. Konishi T. Developmental and activity-dependent changes in $\mathrm{K}+$ currents in satellite glial cells in mouse superior cervical ganglion. Brain Res. 1996;708(1-2):7-15.

30. Lecca D, Ceruti S, Fumagalli M, Abbracchio MP. Purinergic trophic signalling in glial cells: functional effects and modulation of cell proliferation, differentiation, and death. Purinergic Signal. 2012;8(3):539-557.

31. Hanani M. Satellite glial cells in sensory ganglia: from form to function. Brain Res Brain Res Rev. 2005;48(3):457-476.

32. Ohara PT, et al. Gliopathic pain: when satellite glial cells go bad. Neuroscientist. 2009;15(5):450-463.

33. Hanani M, Caspi A, Belzer V. Peripheral inflammation augments gap junction-mediated coupling among satellite glial cells in mouse sympathetic ganglia. Neuron Glia Biol. 2010;6(1):85-89.

34. Ajijola OA, Vaseghi M, Mahajan A, Shivkumar K. Bilateral cardiac sympathetic denervation: why, who and when? Expert Rev Cardiovasc Ther. 2012;10(8):947-949.

35. Vaseghi M, et al. Cardiac sympathetic denervation in patients with refractory ventricular arrhythmias or electrical storm: intermediate and long-term follow-up. Heart Rhythm. 2014;11(3):360-366.

36. Pêgo-Fernandes PM, et al. Endoscopic left sympathetic blockade in the treatment for dilated cardiomyopathy. Arq Bras Cardiol. 2010;95(6):685-690.

37. Ajijola OA, et al. Focal myocardial infarction induces global remodeling of cardiac sympathetic innervation: neural remodeling in a spatial context. Am J Physiol Heart Circ Physiol. 2013;305(7):H1031-H1040 